\title{
Una Práctica de Laboratorio Innovadora: Crecimiento y Caracterización de ZnS Puro y Dopado con Fe ${ }^{2+}$
}

\author{
Rosario Soriano, Julián González, Mariano Pellizzer y Cristina Di Stefano \\ Universidad Tecnológica Nacional, Facultad Regional Buenos Aires, Departamento de Ingeniería \\ Química, Medrano 951, Buenos Aires-Argentina (e-mail: mrs@secyt.frba.utn.edu.ar)
}

\begin{abstract}
Resumen
El trabajo consiste en una práctica de laboratorio de Química Inorgánica, para estudiantes de Ingeniería. Se preparan muestras de ZnS y ZnS dopado con Fe. En el caso de ZnS los precursores son $\mathrm{Na}_{2} \mathrm{~S}$ y $\mathrm{ZnCl}_{2}$. La muestra se dopa con solución saturada de $\mathrm{FeCl}_{2}$. ZnS tiene un ancho de banda prohibido de $3,68 \mathrm{eV}$, una fuerte fluorescencia y presenta resistencia a un alto campo eléctrico por lo que se trata de un material con aplicaciones interesantes para celdas solares, ventanas de IR, láseres y sensores. La difracción de RX evidencia la estructura nanocristalina. Los difractogramas muestran la presencia de cada componente y la estructura de la muestra. Se analizan también las propiedades magnéticas y esto permite detectar electrones desapareados. Los estudiantes desarrollan una técnica de síntesis y realizan un trabajo de investigación para analizar las propiedades y compararlas, recurriendo a equipos y técnicas diferentes.
\end{abstract}

Palabras clave: nanocristales, difractogramas, propiedades magnéticas, prácticas de laboratorio

\section{An innovating Laboratory Experiment: Characterization and Growth of Pure ZnS and $\mathrm{Fe}^{2+}$ Doped ZnS}

\begin{abstract}
This work consists on a laboratory experience for Inorganic Chemistry, for Engineer students. Samples of $\mathrm{ZnS}$ and $\mathrm{ZnS}$ doped with $\mathrm{Fe}$ are prepared. For $\mathrm{ZnS}$ case the precursors are $\mathrm{Na}_{2} \mathrm{~S}$ and $\mathrm{ZnCl}_{2}$. The sample is doped with $\mathrm{FeCl}_{2}$ saturated solution. $\mathrm{ZnS}$ has a band gap of $3,68 \mathrm{eV}$, presents a strong fluorescence and is resistant to an electric high field, it is a material with interesting applications for solar cells, IR windows, lasers and sensors. The X-ray diffraction evidences the nanocrystal structure. Diffractograms show the presence of each component and the structure of the sample. Magnetic properties are also studied which allow to detect odd electrons. The students develop a synthesis technique and they perform a research work to analyze properties and to compare them, using different equipments and techniques.
\end{abstract}

Keywords: nanocrystals, diffractograms, magnetic properties, laboratory experience 


\section{INTRODUCCIÓN}

Este trabajo fue realizado por un grupo de alumnos becarios, bajo la supervisión de los docentes a cargo. Se trata de una práctica de laboratorio que surge a partir del trabajo de un grupo de investigación y tiene aplicación en una materia de grado. Este tipo de transferencia de conocimientos es muy importante en la articulación de dos actividades primordiales del ámbito universitario: la docencia y la investigación.

Distintos autores (Abraham, 2005; Bransford et al., 1999) han estudiado cómo el compromiso activo y la interacción con otros compañeros son esenciales para los estudiantes para lograr una verdadera comprensión y retención. Se ha investigado (Cooper et al., 2009) y se sabe que los estudiantes que trabajan en equipos de aprendizaje aprenden más; entienden más; recuerdan más; se sienten mejor consigo mismos y con los demás; tienen actitudes más positivas con respecto del área de estudio, el curso, y los docentes; y adquieren habilidades de pensamiento crítico, estrategias de aprendizaje cognitivo, y otras habilidades procesales que son esenciales para su desarrollo como aprendices independientes (Sabelli, 2008 y Cooper, 2005). Según la experiencia recogida por investigadores que implementaron innovaciones en temáticas y metodologías de enseñanza en ciencias aplicadas (Barab et al., 2000 y Labarrere y Quintanilla, 2002) esta forma de trabajo ayuda a los alumnos a desarrollar su propia estrategia de aprendizaje y no recibirla impuesta por un tercero. La tarea que se realizó y que se propone en este trabajo presenta fuertes ventajas en el aprendizaje de los temas tratados. Para realizar esta práctica los estudiantes preparan las muestras y luego realizan sobre ellas una serie de estudios que les permiten, no sólo desarrollar distintas técnicas de análisis, sino también incorporar la información que dichas técnicas les dan. El análisis y trabajo con los datos y los difractogramas obtenidos les permiten realizar un estudio muy completo.

El sulfuro de cinc ( $\mathrm{ZnS}$ ) es una sal fluorescente y brilla cuando le inciden rayos ultravioletas y radiación alfa, beta o gamma. Es un compuesto semiconductor de la familia II-VI, tipo n. Como nanocristal, posee la particularidad que su ancho de banda aumenta cuando disminuye el tamaño de partícula, debido a los efectos del confinamiento cuántico, según Fernandez Sanchez (2004). Las nanopartículas constituyen un estado intermedio entre las estructuras moleculares y los materiales cristalinos masivos. El ZnS, empleado como punto cuántico (QD) es un material de banda directa y fuerte fluorescencia, con importantes aplicaciones tecnológicas en una amplia gama de dispositivos optoelectrónicos: detectores de infrarrojo, diodos de emisión de luz (LED) en la longitud del azul y el verde, celdas solares, según Kumbhojkar et al. (2000) y Angelo y Farnood (2009). Estos materiales siguen siendo los más utilizados en la fabricación de detectores de IR de alto desempeño por dos propiedades relevantes: gran velocidad de respuesta (nanosegundos) y posibilidad de variar su rango de respuesta espectral con la composición.

\section{METODOLOGÍA}

En esta sección se describe la preparación de las muestras, trabajo que fue realizado enteramente por los estudiantes. Las muestras nanocristalinas se obtuvieron por precipitación. En el caso de ZnS, siguiendo a Motlan et al. (2007), los precursores son soluciones de $\mathrm{Na}_{2} \mathrm{~S}$ y $\mathrm{ZnCl}_{2}$. En el caso de la muestra dopada a la solución de $\mathrm{ZnCl}_{2}$ se le agrega, con agitación continua, solución saturada de $\mathrm{FeCl}_{2}$, siguiendo a González Beermann et al. (2004). El procedimiento seguido para la obtención de los cristales puros es el siguiente: $100 \mathrm{~mL}$ de solución 1,5 M de $\mathrm{ZnCl}_{2}$ se colocan en un vaso de precipitado, sobre un calefactor eléctrico, provisto de agitador magnético, Kumbhojkar et al. (2000). A continuación se agrega, lentamente y manteniendo la agitación constante, $100 \mathrm{~mL}$ de solución $1,5 \mathrm{M}$ de $\mathrm{Na}_{2} \mathrm{~S}$, Kortan et al. (1990). La temperatura se mantiene en $50{ }^{\circ} \mathrm{C}$, aproximadamente. Se observa que la solución se enturbia, según va produciéndose la precipitación de los cristales. Concluida la reacción, se ajusta el $\mathrm{pH}$ a un valor de 9 , mediante la adición de solución $0,1 \mathrm{M}$ de $\mathrm{NaOH}$. Este valor de $\mathrm{pH}$ es el óptimo para obtener la precipitación de partículas nanométricas de $\mathrm{ZnS}$. Si se excede este valor precipita $\mathrm{ZnO}$ y $\mathrm{Zn}(\mathrm{OH})_{2}$ (Motlan et al., 2007). Se suspende la agitación y la calefacción, y se permite la decantación de los cristales a temperatura ambiente. Se descarta la solución sobrenadante y se efectúa el lavado de los cristales mediante el agregado de pequeñas porciones de agua destilada y posterior separación mediante centrifugado. Finalmente se recoge el precipitado sobre un vidrio de reloj y se seca en estufa a $120^{\circ} \mathrm{C}$ hasta eliminar todo resto de humedad. Para efectuar el dopado con Fe 
(González Beermann et al., 2004), el procedimiento descrito anteriormente se modifica de la siguiente manera: a la solución de $\mathrm{ZnCl}_{2}$, sometida a calefacción y bajo agitación continua, se le agregan $5 \mathrm{~mL}$ de solución saturada de $\mathrm{FeCl}_{2}$. Se deja estabilizar la mezcla 15 minutos y a continuación se opera como en la obtención del ZnS puro.

\section{Caracterización}

Los cristales se caracterizan mediante difracción de RX y EDAX, empleando un microscopio electrónico. Los equipos en este estudio fueron: para RX un Philips PW1830 y para el EDAX un Philips XL30ESEM.
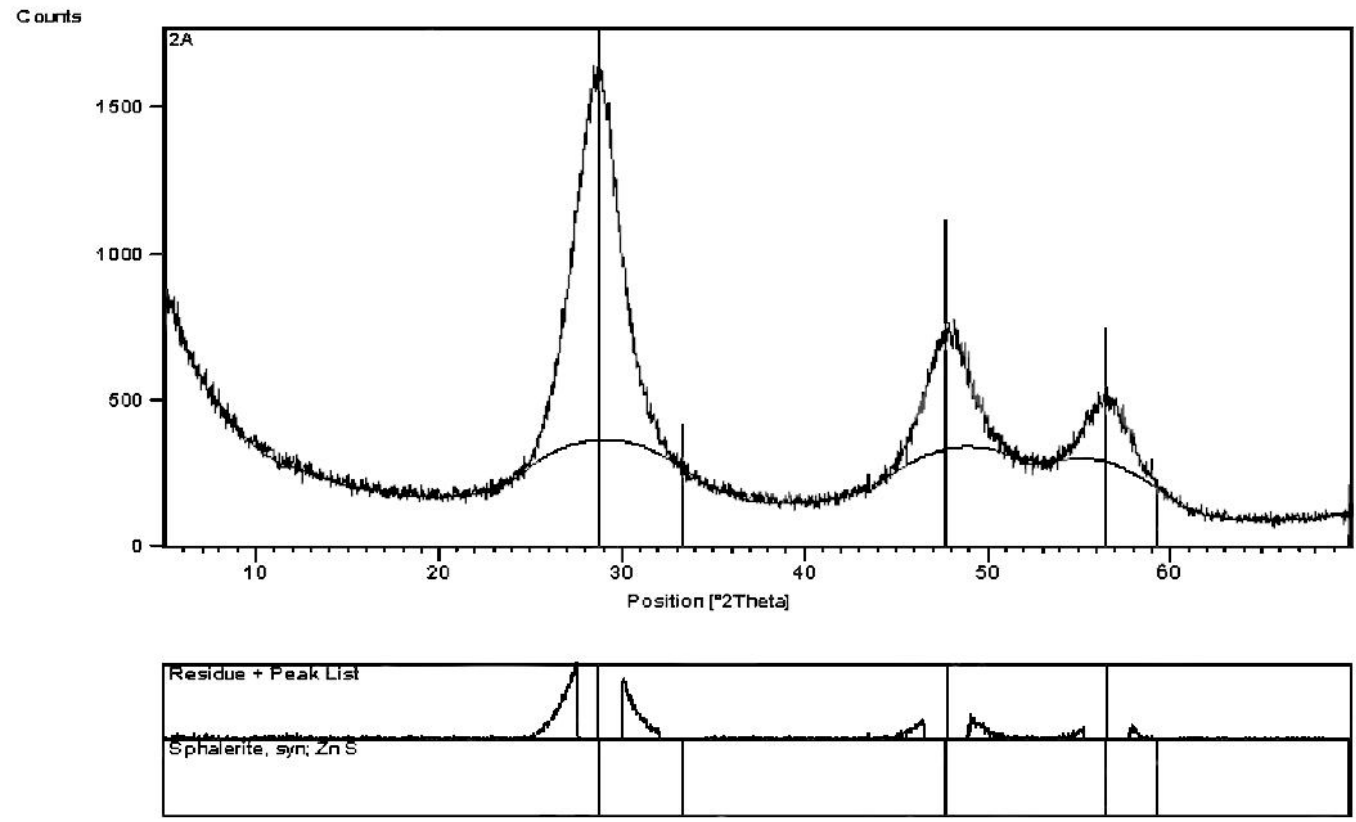

Fig. 1. RX del ZnS puro

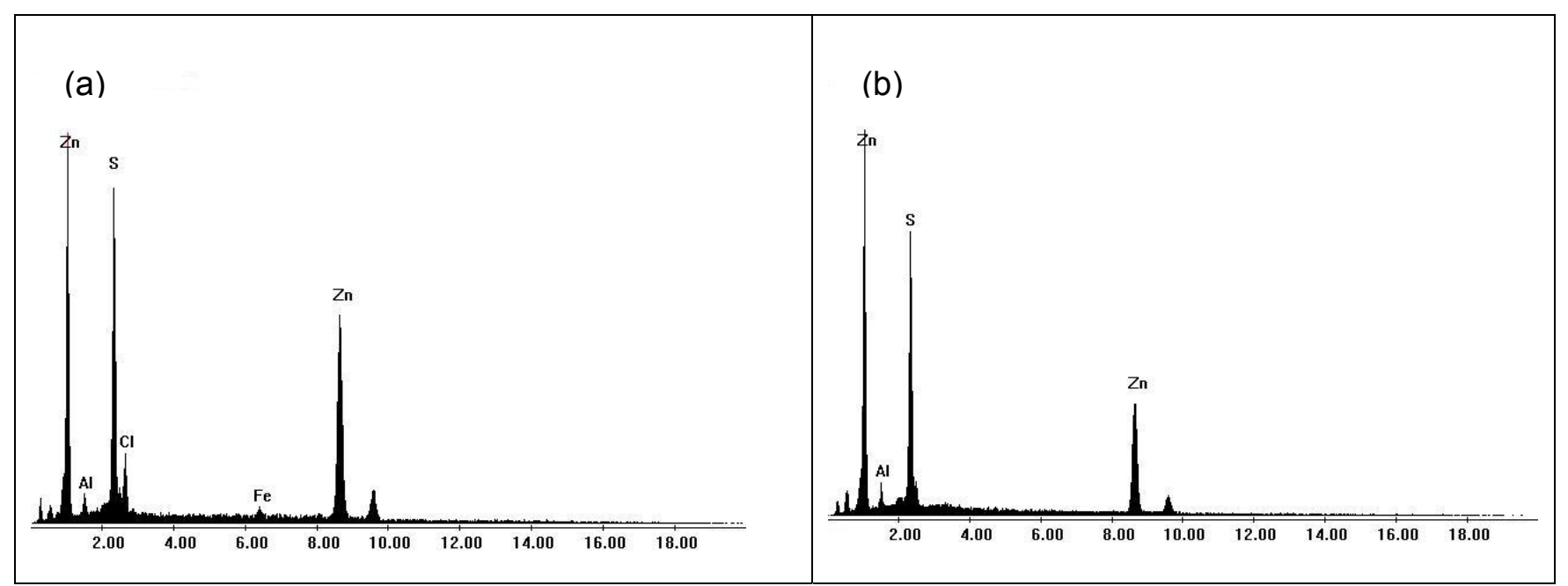

Fig. 2. EDAX de la muestra dopada (a) y de la muestra pura (b)

El tamaño del cristal se obtiene del difractograma, aplicando la ecuación de Sherrer (1918). Los resultados dieron $10 \mathrm{~nm}$, el equipo utilizado puede dar este resultado en forma directa y también se lo puede calcular. La muestra de ZnS puro y la muestra dopada con Fe dan el mismo patrón de difracción de RX ya que la presencia de Fe es pequeña y no se detecta.

El difractograma inferior de la figura 1 corresponde a los valores de referencia para ZnS y se verifica una muy buena coincidencia entre ese espectro y el experimental, lo que prueba que se obtuvo ZnS puro.En la figura 2 aparecen los EDAX de la muestra pura y la muestra dopada. Este 
método de determinación, que es más sensible, puede verificar la presencia del dopante aunque esté presente en pequeñas cantidades. El pico de Al que aparece en ambos difractogramas se puede deber al portamuestra ya que es constante en ambas. La pequeña cantidad de $\mathrm{Cl}$ que aparece en la muestra dopada se puede deber a un lavado ineficiente de la muestra.

\section{Propiedades magnéticas}

Se mide susceptibilidad magnética y no momentos magnéticos directamente, y a partir de estas medidas se calcula el momento magnético. Es a partir de una correcta interpretación de los valores medidos de los momentos magnéticos (Bates, 1961), que se obtiene información química útil.

En este trabajo se utiliza una balanza analítica adaptada, que permite registrar la masa de la muestra en ausencia de campo magnético y en presencia del mismo. La diferencia de masa entre estas dos situaciones se relaciona matemáticamente con la fuerza resultante de la interacción entre el campo magnético externo (que se logra con un electroimán) y el inducido, que es el que produce la muestra. Para poder registrar diferencias de masa razonables, se necesita usar una masa del orden de los gramos y potentes electroimanes refrigerados, así como una balanza analítica modificada. La que se usó en este trabajo es la que utiliza el método alternativo de Gouy, ideado por Evans (1974) del Imperial College de Londres.

En lugar de medir la fuerza que experimenta la muestra en presencia del campo magnético, se mide la acción de un pequeño imán permanente adosado a un sistema basculante, similar al de una balanza de platillos. En el otro brazo del sistema hay una bobina, la interacción de esta bobina con el segundo imán permanente genera una oposición al movimiento del primero, con lo que se registra una corriente que circula por una bobina del electroimán. La posición del equilibrio del fiel se consigue cuando esta fuerza compensa la interacción imán permanente-muestra. Así esa corriente es directamente proporcional a la acción que ejerce la sustancia sobre el imán permanente móvil.

La susceptibilidad por gramo de sustancia se obtiene con la ecuación:

$X_{g}=C \cdot I \cdot\left(R-R_{0}\right) /\left(10^{9} \mathrm{~m}\right)(\mathrm{cgs})$

(1)

En esta ecuación los parámetros que aparecen son:

C: es la constante de la balanza (constante de proporcionalidad que se obtiene por calibración)

I: es la longitud de la muestra en el tubo (en $\mathrm{cm}$, mínimo se deben colocar $2,5 \mathrm{~cm}$ )

$\mathrm{R}$ : es la corriente que circula con la muestra

$\mathrm{R}_{0}$ : es la corriente que circula sin la muestra (se coloca como blanco un tubo vacío)

Mr: es la masa de muestra empaquetada en el tubo de medida.

Para obtener el momento magnético en magnetones de Bohr se aplica la ecuación que sigue:

$\mu=2,828\left(X_{g} \cdot \mathrm{Mr} \cdot \mathrm{T}\right)^{1 / 2}$

(2)

donde $\mathrm{T}=$ Temperatura absoluta $(\mathrm{K})$.

Existe una relación entre el número de electrones desapareados y el momento magnético, la que se presenta en la siguiente ecuación:

$\mu=(n(n+2))^{1 / 2}$

(3)

Tabla 1: Datos de la muestra

\begin{tabular}{|c|c|c|c|}
\hline Compuesto & Metal & Configuración & Masa \\
\hline ZnS & & & 0,2227 \\
\hline ZnS: Fe & $\mathrm{Fe}^{+2}$ & $\mathrm{~d}^{6}$ & 0,2397 \\
\hline
\end{tabular}


A partir de las medidas realizadas se puede calcular el número de electrones desapareados de cada sustancia. A partir de este dato se puede determinar la masa de hierro incorporada ya que los electrones desapareados del Fe son los responsables del momento magnético. El ZnS, como se verifica en la experiencia, es diamagnético (ya que es de capa completa).

En las tablas 1 y 2 se presentan los datos, las medidas realizadas y los cálculos correspondientes.

Tabla 2: Resultados

\begin{tabular}{|c|c|c|c|c|c|c|c|c|}
\hline Compuesto & $\mathrm{Mr}$ & $\mathrm{R}_{0}$ & $\mathrm{R}$ & $\mathrm{R}_{0}-\mathrm{R}$ & $\mathrm{I}$ & $X$ & $\mu$ & $\mathrm{n}$ \\
\hline ZnS & 97,43 & -062 & -080 & -18 & 3,1 & diamagnético & & 0 \\
\hline ZnS: $\mathrm{Fe}$ & 97,33 & -031 & 13 & 44 & 3,3 & $6,02310^{-7}$ & 0,3736879 & 0,0675404 \\
\hline
\end{tabular}

\section{RESULTADOS Y DISCUSIÓN}

La práctica desarrollada se implementa a través de una transferencia de conocimientos desde un grupo de investigación hacia la carrera de grado. Es fundamental que el alumnado interprete que no se trata de módulos estancos. Según sea el área de investigación que está desarrollando, los conceptos y destrezas específicas que adquiere tienen una potencial aplicación en las materias de grado, redundando en un enriquecimiento de las mismas.

En esta actividad se cumple este objetivo. Los alumnos, pertenecientes a los grupos de investigación en materiales, crecieron y caracterizaron ZnS puro y dopado y corroboraron los efectos del dopado sobre las propiedades magnéticas del mismo. Tuvieron acceso a las técnicas específicas a partir de su actividad dentro del grupo de investigación. A partir de su trabajo pudo implementarse una práctica de laboratorio al alcance de los estudiantes que cursan la materia Química Inorgánica, correspondiente al segundo nivel de la carrera Ingeniería Química. Este tipo de actividades permitirá más adelante implementar prácticas de laboratorio con los estudiantes en materias de grado. El equipo que se utilizó es sencillo y no es costoso.

En todo momento los alumnos participaron de la toma de datos y medidas, si bien la caracterización que requirió equipos como el de RX y EDAX, estuvo a cargo de operadores especializados, ellos estaban presentes y discutieron los resultados.

El grupo de alumnos, cinco estudiantes becados que trabajan con el equipo de investigación en materiales, realizó parte del trabajo bajo la supervisión de los investigadores y otra parte de forma independiente de modo de completar un informe con los datos que aparecen en el presente trabajo. Esta tarea los enfrentó con un trabajo en grupo con discusiones y acuerdos, entre ellos y con los investigadores, para realizar el análisis y la presentación de los resultados.

Este trabajo permitió al grupo de estudiantes efectuar las actividades prácticas usuales en un laboratorio químico: ajuste de $\mathrm{pH}$, separación de fases, lavados reiterados, secado, etc. Los métodos de caracterización empleados, si bien fueron efectuados en equipos complejos, a cargo de operadores capacitados, pudieron ser interpretados por el grupo con la ayuda de los docentes. La información adicional necesaria, no excede el nivel de formación de los alumnos. Asimilan los conocimientos necesarios y pueden desempeñarse sin inconvenientes. El grupo de estudiantes preparó el informe con los cálculos y la interpretación de los resultados, mostrando una clara comprensión de los temas involucrados, además del entusiasmo. Con esto, el alumno aprende a aprender y alcanza logros que son infrecuentes, tan temprano, en los cursos tradicionales. Los alumnos se muestran así fuertemente motivados, pues los logros son "adictivos". El logro que motiva es aquél que lleva a hacer excepcionalmente bien aquello en lo que ya se es bueno (Drucker, 2002).

\section{CONCLUSIONES}

Mediante las determinaciones analíticas se verifica la obtención de las sustancias buscadas y a través de las propiedades magnéticas se determina que el ZnS es un sistema de capa cerrada mientras que el efecto del dopado da carácter magnético al compuesto. 
El estudio de RX permite determinar el tamaño de las partículas, que resulta ser del orden de 10 $\mathrm{nm}$ por lo que hablamos de nanocristales.

En la evaluación de las propiedades magnéticas, el equipo es muy sencillo y los fundamentos teóricos son conocidos por los alumnos que cursan las materias Química Inorgánica y Física II.

\section{AGRADECIMIENTOS}

Los autores agradecen a la Dra. María dos Santos Afonso por su valiosa colaboración.

\section{REFERENCIAS}

Abraham, M.R.; Inquiry and the learning cycle approach. In Chemists' guide to effective teaching by Pienta, N.J., M.M. Cooper, and T.J. Greenbowe, Upper Saddle River, NJ: Prentice Hall (2005).

Angelo P.D. y R.R. Farnood; Chapter 8: Inkjet ink containing inorganic doped semiconductor (ZnS:Mn) nanoparticle pigments. In Nanotechnology: Biofuels, Renewable Energy, Coatings, Fluidics and Compact Modeling ISBN: 978-1-4398-1784-1 (2009).

Barab, S.A., Hay, K.E., M. Barnett y T. Keating; Virtual solar system Project: Building Understanding through model Building. Journal of Rechearch in Science Teaching: 37, 719-756 (2000).

Bates L.F.; Modern Magnetism, University Press, Cambridge (1961).

Bransford, J.D., A.L. Brown y R.R. Cocking; How People Learn. Washington, DC: National Academy Press (1999).

Cooper M., T. Greenbow y N. Pienta; Chemists' guide to effective teaching, Prentice Hall (2009).

Cooper, M.M.; An introduction to small-group learning. In Chemists' guide to effective teaching by M. M. Cooper, T.J. Greenbow and N. Pienta, Prentice Hall (2005).

Drucker Peter F.; Escritos fundamentales: Tomo 3, La sociedad, $1^{\mathrm{a}}$ Ed., Buenos Aires: Ed. Sudamericana (2002).

Evans F.; A new type of magnetic balance, J. Phys. E: Sci. Instrum.: 7, 247-249 (1974).

Fernández Sánchez, P. Materiales optoelectrónicos. Semiconductores II-VI (2204), http://material.fis.ucm.es/ paloma/private/notas/aplicaciones/NANOCRISTALES.pdfAcceso julio $\underline{2009}$

González Beermann P.A., B.R. McGarvey, S. Muralidharan y R.C.W. Sung; EPR Spectra of $\mathrm{Mn}^{2+}-$ Doped ZnS Quantum Dots. Chem. Mater.: 16, 915-918 (2004).

Kortan A.R. y otros cinco autores; Nucleation and growth of cadmium selendie on zinc sulfide quantum crystallite seeds, and vice versa, in inverse micelle media. J. Am. Chem. Soc.: 112, 13271332 (1990).

Kumbhojkar N., V.V. Nikesh, A. Kshirsagar y S. Mahamuni; Photophysical Properties of ZnS Nanoclusters. J. Appl. Phys.: 88, 6260 (2000)

Labarrere, A. y M. Quintanilla; La solución de problemas científicos en el aula. Reflexiones desde los planos de análisis y desarrollo de estudiantes de ciencias. Revista pensamiento educativo: 30 , 121-138 (2002).

Motlan, Guanghua Zhu, y otros cuatro autores; Anneling of ZnS nanocrystals grow by colloidal synthesis. Optical Materials: $29,1579-1583$ (2007).

Sabelli, N.; Alfabetización en el Siglo XXI: Ciencia y tecnología al alcance de todos, Proyecciones UTN: 6(2), ISSN 1667-8400 (2008).

Scherrer P.; Göttinger Nachrichten Gesell., 2, 98 (1918). 